\title{
The first orbital elements for eight binaries
}

\author{
G.M. Popović, R. Pavlović, and V. Živkov \\ Astronomical Observatory, Volgina 7, 11160 Belgrade 74, Yugoslavia \\ e-mail: gpopovic@aob.bg.ac.yu
}

Received April 12, 1999; accepted February 25, 2000

\begin{abstract}
The orbital elements, masses and orbital parallaxes are communicated for the first time for the following eight double stars: WDS 04089+4614 = ADS $3007=$ A $998=$ HD 25987, WDS $04275+1113=\operatorname{ADS} 3228=$ BU $1186=$ HD 28217, WDS 04400+2301 = ADS $3370=$ HU $442=$ HD 29538, WDS 18018+0118 = ADS 10990 $\mathrm{AB}=\mathrm{BU} 1125 \mathrm{AB}=\mathrm{HD} 164577$, WDS $18033+3921=$ ADS $11023=$ STF $2275=$ HD 88432, WDS $18054+6216=$ ADS $11073=$ HU $1290=$ HD 166206, WDS 19389+3514 $=\operatorname{ADS} 12746=\mathrm{HU} 953=\mathrm{HD} 185696$, WDS $20176+2622$ $=\operatorname{ADS} 13649=\mathrm{BU} 984=\mathrm{HD} 193095$.
\end{abstract}

Key words: astrometry — binaries: visual

\section{Introduction}

The orbital elements for double stars A 998, BU 1186 , HU 442, BU 1125, STF 2275, HU 1290, HU 953, BU 984 have not been derived as yet. In spite of significant uncertainties in their distances, usual in the case of the visual measurements, the observed arcs allow a sufficiently reliable determination of their elliptical orbits.

The orbital elements are derived by using the procedure proposed by Popović \& Pavlović (1995), the absolute magnitudes of the components, the masses and parallaxes are obtained by applying Angelov's (1993) relations, and the errors of the fitting parameters are obtained by using the Eichhorn \& Xu algorithm (1990). The elements are given for the epoch J2000.0. All elements have been published in the "Information Circular 135 and 136, IAU, Comm. 26".

The orbital elements and the corresponding astrophysical quantities are given in Table 1 , the $\mathrm{O}-\mathrm{C}$ measurements in Table 2. The authors of the orbits are given at the bottom of Table 1 . The ephemeris are calculated for
Table 1. Orbital elements, absolute magnitudes, masses and parallaxes

\begin{tabular}{|c|c|c|c|c|}
\hline Name & A 998 & BU 1186 & HU 442 & $\mathrm{BU} 1125 \mathrm{AB}$ \\
\hline WDS & $04089+4614$ & $04275+1113$ & $04400+2301$ & $18018+0118$ \\
\hline ADS & 3007 & 3228 & 3370 & 10990 \\
\hline HD & 25987 & 28217 & 29538 & 164577 \\
\hline$m$ & $7.7-8.0$ & $6.0-8.0$ & $9.3-9.9$ & $4.4-9.2$ \\
\hline Sp. & A0V & B8IV & A2 & $\mathrm{A} 2 \mathrm{Vn}$ \\
\hline \multirow[t]{2}{*}{$P(\mathrm{yr})$} & 158.245 & 410.671 & 248.149 & 264.378 \\
\hline & \pm 7.794 & \pm 67.796 & \pm 55.058 & \pm 40.621 \\
\hline \multirow{2}{*}{$n\left(^{\circ} / \mathrm{yr}\right)$} & 2.2749 & 0.8766 & 1.4507 & 1.3617 \\
\hline & \pm 0.1120 & \pm 0.1445 & \pm 0.3219 & \pm 0.2092 \\
\hline \multirow[t]{2}{*}{$T$} & 2010.16 & 1948.17 & 1988.71 & 2070.67 \\
\hline & \pm 2.03 & \pm 3.71 & \pm 10.07 & \pm 19.19 \\
\hline \multirow{2}{*}{$a\left({ }^{\prime \prime}\right)$} & 0.2814 & 0.7736 & 0.4537 & 0.7974 \\
\hline & \pm 0.0103 & \pm 0.0770 & \pm 0.0614 & \pm 0.0315 \\
\hline \multirow[t]{2}{*}{$e$} & 0.6922 & 0.2104 & 0.1294 & 0.2642 \\
\hline & \pm 0.0522 & \pm 0.0856 & \pm 0.0532 & \pm 0.1390 \\
\hline \multirow[t]{2}{*}{$i\left(^{\circ}\right)$} & 123.76 & 102.16 & 69.34 & 53.29 \\
\hline & \pm 2.18 & \pm 0.40 & \pm 0.84 & \pm 1.87 \\
\hline \multirow[t]{2}{*}{$\Omega\left({ }^{\circ}\right)$} & 170.76 & 170.23 & 177.93 & 7.10 \\
\hline & \pm 3.17 & \pm 0.45 & \pm 0.46 & \pm 5.25 \\
\hline \multirow{2}{*}{$\omega\left(^{\circ}\right)$} & 46.91 & 27.19 & 259.92 & 232.48 \\
\hline & \pm 5.84 & \pm 4.73 & \pm 21.05 & \pm 7.68 \\
\hline$A\left({ }^{\prime \prime}\right)$ & -0.17142 & -0.66548 & 0.08503 & -0.43521 \\
\hline$B\left({ }^{\prime \prime}\right)$ & 0.14357 & 0.19014 & 0.15460 & -0.43521 \\
\hline$\left.F^{\prime \prime \prime}\right)$ & 0.21999 & 0.37295 & -0.44539 & 0.66351 \\
\hline$G\left({ }^{\prime \prime \prime}\right)$ & 0.07244 & 0.08281 & 0.04411 & -0.20988 \\
\hline$C\left(^{\prime \prime \prime}\right)$ & \pm 0.17086 & \pm 0.34556 & $\mp 0.41798$ & $\mp 0.50704$ \\
\hline$H\left({ }^{\prime \prime \prime}\right)$ & \pm 0.15984 & \pm 0.67266 & $\mp 0.07429$ & $\mp 0.38937$ \\
\hline$T_{\mathrm{u}}(\mathrm{yr})$ & 2007.18 & 1928.13 & 2047.49 & 2144.45 \\
\hline$T_{\mathrm{s}}(\mathrm{yr})$ & 2031.86 & 2107.92 & 1943.48 & 2047.73 \\
\hline$M_{\mathrm{A}}(\mathrm{mag})$ & 1.7 & 0.7 & 4.0 & -0.2 \\
\hline$M_{\mathrm{B}}(\mathrm{mag})$ & 2.0 & 3.6 & 4.6 & 4.6 \\
\hline $\mathfrak{M}_{\mathrm{A}}(\odot)$ & 1.9 & 2.4 & 1.1 & 3.0 \\
\hline $\mathfrak{M}_{\mathrm{B}}(\odot)$ & 1.8 & 1.3 & 1.0 & 1.0 \\
\hline$\pi\left({ }^{\prime \prime}\right)$ & 0.006 & 0.009 & 0.009 & 0.012 \\
\hline Author & Pavlović & $\begin{array}{l}\text { Popović \& } \\
\text { Pavlović }\end{array}$ & $\begin{array}{c}\text { Pavlović \& } \\
\text { Živkov }\end{array}$ & $\begin{array}{l}\text { Popović \& } \\
\text { Živkov }\end{array}$ \\
\hline
\end{tabular}


Table 1. continued

\begin{tabular}{|c|c|c|c|c|}
\hline Name & STF 2275 & HU 1290 & HU 953 & BU 984 \\
\hline WDS & $18033+3921$ & $18054+6216$ & $19389+3514$ & $20176+2622$ \\
\hline ADS & 11023 & 11073 & 12746 & 13649 \\
\hline HD & 88432 & 166206 & 185696 & 193095 \\
\hline$m$ & $9.3-9.5$ & $9.2-9.5$ & $8.8-9.2$ & $9.0-9.3$ \\
\hline Sp. & G0 & F8 & G0 & F8 \\
\hline \multirow[t]{2}{*}{$P(\mathrm{yr})$} & 525.460 & 294.940 & 300.8386 & 286.5759 \\
\hline & \pm 22.316 & \pm 10.030 & \pm 41.540 & \pm 28.928 \\
\hline \multirow[t]{2}{*}{$n\left({ }^{\circ} / \mathrm{yr}\right)$} & 0.6851 & 1.2206 & 1.19666 & 1.2562 \\
\hline & \pm 0.0291 & \pm 0.0415 & \pm 0.1652 & \pm 0.1268 \\
\hline \multirow[t]{2}{*}{$T$} & 2012.872 & 1985.026 & 2009.729 & 2080.014 \\
\hline & \pm 42.54 & \pm 44.81 & \pm 4.97 & \pm 9.62 \\
\hline \multirow{2}{*}{$a\left({ }^{\prime \prime}\right)$} & 0.6786 & 0.4755 & 0.7364 & 0.8000 \\
\hline & \pm 0.2007 & \pm 0.0528 & \pm 0.1963 & \pm 0.0523 \\
\hline \multirow[t]{2}{*}{$e$} & 0.4767 & 0.2567 & 0.4491 & 0.0885 \\
\hline & \pm 0.0339 & \pm 0.0514 & \pm 0.0450 & \pm 0.0774 \\
\hline \multirow[t]{2}{*}{$i\left(^{\circ}\right)$} & 77.95 & 120.35 & 54.70 & 70.19 \\
\hline & \pm 10.16 & \pm 0.48 & \pm 4.05 & \pm 7.45 \\
\hline \multirow[t]{2}{*}{$\Omega\left(^{\circ}\right)$} & 131.60 & 110.45 & 8.52 & 43.99 \\
\hline & \pm 2.84 & \pm 6.40 & \pm 8.58 & \pm 3.16 \\
\hline \multirow[t]{2}{*}{$\omega\left(^{\circ}\right)$} & 142.76 & 80.56 & 314.66 & 2.98 \\
\hline & \pm 76.34 & \pm 12.50 & \pm 42.04 & \pm 14.60 \\
\hline$A\left({ }^{\prime \prime}\right)$ & 0.29367 & 0.19481 & 0.55672 & 0.56502 \\
\hline$B\left({ }^{\prime \prime}\right)$ & -0.46149 & 0.15584 & -0.22269 & 0.56502 \\
\hline$F\left({ }^{\prime \prime}\right)$ & 0.35655 & 0.20080 & 0.47373 & -0.21789 \\
\hline$G\left({ }^{\prime \prime}\right)$ & -0.23298 & -0.42572 & 0.37341 & 0.16591 \\
\hline$\left.C^{(\prime \prime}\right)$ & $\mp 0.40168$ & \pm 0.40478 & $\mp 0.42750$ & \pm 0.03907 \\
\hline$H\left({ }^{\prime \prime}\right)$ & $\mp 0.52835$ & \pm 0.06727 & $\mp 0.42241$ & \pm 0.75167 \\
\hline$T_{\mathrm{u}}(\mathrm{yr})$ & 1870.48 & 1941.82 & 2023.48 & 2078.04 \\
\hline$T_{\mathrm{s}}(\mathrm{yr})$ & 2030.61 & 2042.22 & 1936.30 & 2220.49 \\
\hline$M_{\mathrm{A}}(\mathrm{mag})$ & 3.8 & 3.7 & 4.3 & 4.8 \\
\hline$M_{\mathrm{B}}(\mathrm{mag})$ & 4.0 & 4.0 & 4.7 & 5.1 \\
\hline $\mathfrak{M}_{\mathrm{A}}(\odot)$ & 1.2 & 1.2 & 1.1 & 1.0 \\
\hline $\mathfrak{M}_{\mathrm{B}}(\odot)$ & 1.2 & 1.1 & 1.0 & 0.9 \\
\hline$\pi\left({ }^{\prime \prime}\right)$ & 0.008 & 0.008 & 0.013 & 0.015 \\
\hline Author & Popović & $\begin{array}{l}\text { Živkov \& } \\
\text { Pavlović }\end{array}$ & $\begin{array}{l}\text { Živkov \& } \\
\text { Popović }\end{array}$ & $\begin{array}{c}\text { Pavlović \& } \\
\text { Popović }\end{array}$ \\
\hline
\end{tabular}

the next 10 years, beginning with 1999, the step length is one year.

The final part contains the figures with the orbits.

\section{Comments}

Notes to individual binary systems follow, sorted in order of WDS designation (the 2000-epoch right ascensionand declination-based designation used in the Washington Visual Double Star Catalog of Worley \& Douglass 1996).

WDS 04089+4614 = ADS $3007=$ A $998=$ HD 25987 For the purpose of orbit calculating the position angle is corrected for $180^{\circ}$ in four cases as follows: 1981.01, 1982.06, 1983.84, 1985.00.

The obtained orbital elements yield a parallax value in good agreement with the trigonometric parallax given in the Hipparcos Catalogue (0'.00665 \pm 0 0'00096).
The measuring of this pair grows in its difficulty and at the epoch of periastron passage in 2010 the component separation will be $\sim 0$ !'07 only.

WDS 04275+1113 = ADS $3228=$ BU $1186=$ HD 28217 This pair, visible by the naked eye in the constellation of Taurus, was discovered in 1890 by Burnham S.W. The separation of the components was about 0.6 then. From Aitkin's measurement in 1898 the pair grew closer and more unfavourable for the measurements (McAlister \& Hartkopf 1988).

The elements are based on a change in $\theta$ of about $60^{\circ}$, but one should take into account that the pair has a high inclination $\left(i=120^{\circ}\right)$ and that already $1 / 4$ of the orbital plane has been defined by the observations.

For the purpose of obtaining the parallax and absolute magnitude we use the empirical relation concerning the stars above the main sequence in the HR diagram (luminosity class - LC - B8 IV). The Hipparcos Catalogue yields for this pair 0 "'00670 \pm 0 ". 00096 as its trigonometrical parallax, whereas the orbital parallax obtained by us is somewhat higher 0. .'009.

WDS 04400+2301 = ADS $3370=$ HU $442=$ HD 29538 The orbital elements for this Hussey W.L. pair, discovered in 1901, are based on a change in the position angle of $103^{\circ}$. Due to its faint components and the separation not exceeding 0.5 the pair has not been much observed. Nevertheless, the available observations allow to define the orbit position sufficiently well.

The spectral type and the obtained absolute magnitude indicate that the system belongs to LC VI (subdwarfs). By applying the mass-luminosity relation to the stars below the main sequence one obtains a parallax significantly higher $\left(00^{\prime \prime} 009\right)$ than that given in the Tycho Catalogue $\left(00^{\prime \prime} 0016 \pm 0\right.$ ". 0009$)$.

WDS 18018+0118 = ADS $10990=$ BU $1125=$ HD 164577 The orbital elements are obtained from a position-angle change of $104^{\circ}$. The measurements have a large scatter.

The pair is on the main sequence of HR diagram. The obtained orbital parallax 0 .'0012 agrees well with the one given in the Hipparcos Catalogue $\left(0{ }^{\prime \prime} 001231 \pm 0\right.$.'000083).

Popović and Pavlović failed in noticing the duplicity of this system on May 27, 1998 when they tried to measure this pair. The cause is, most probably, in the magnitude difference $\triangle m=4.8$. For this moment, according to the ephemeris, $\rho$ is 0.56 .

WDS $18033+3921=$ ADS $11023=$ STF $2275=$ HD 88432 In the calculation of the elements the position angle is corrected for $180^{\circ}$ in the case of two Baize's observations from 1954.70 and 1956.70 and also for one Worley's observation from 1961.43.

The orbital parallax derived here agrees fully with the trigonometric parallax given in the Hipparcos $(0$.'00836 \pm $0^{\prime \prime}$ 00092). 
0.8

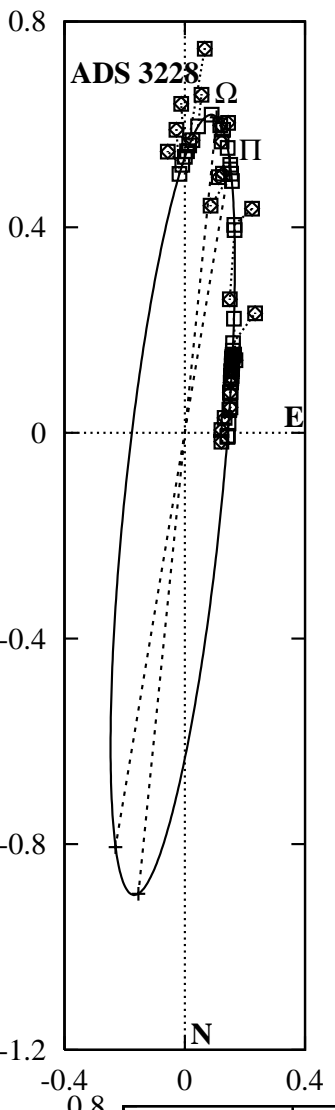

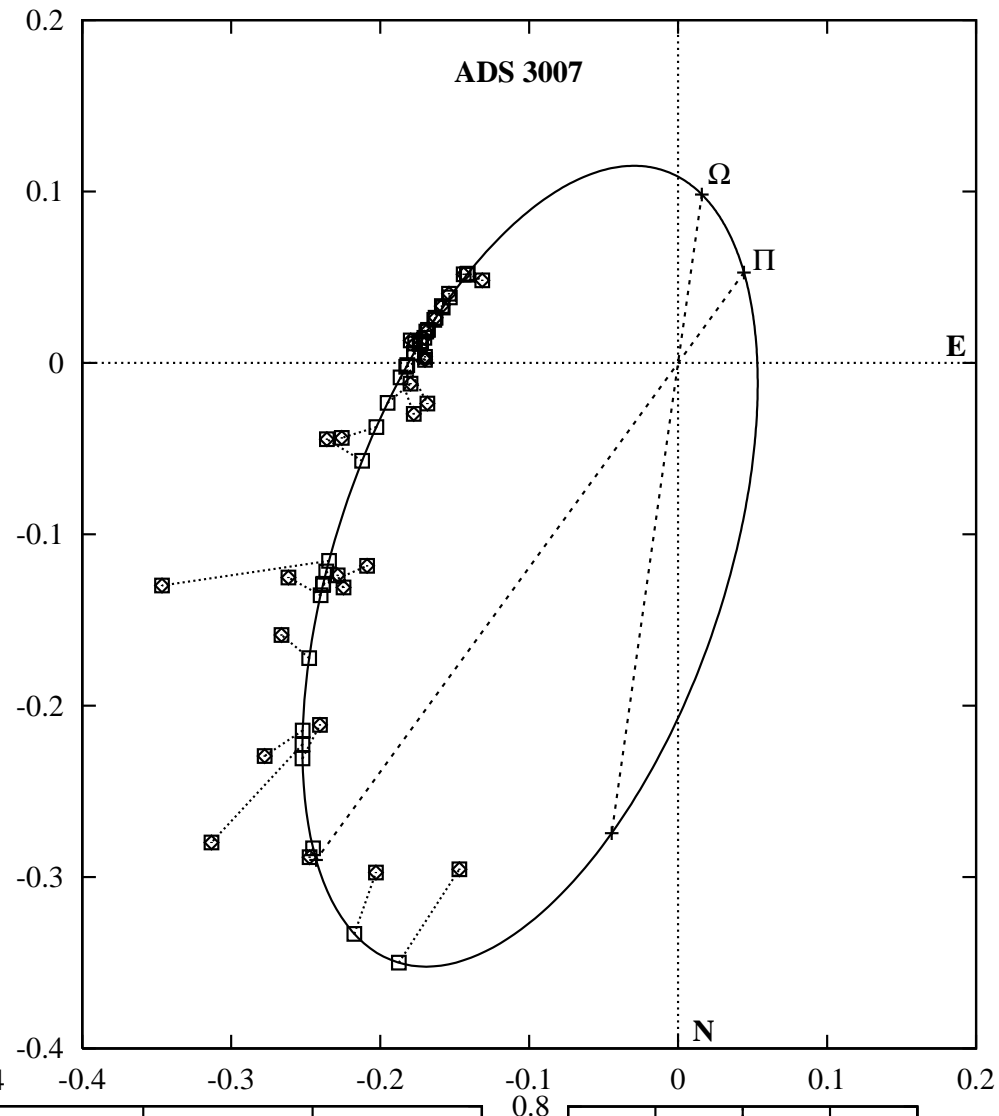
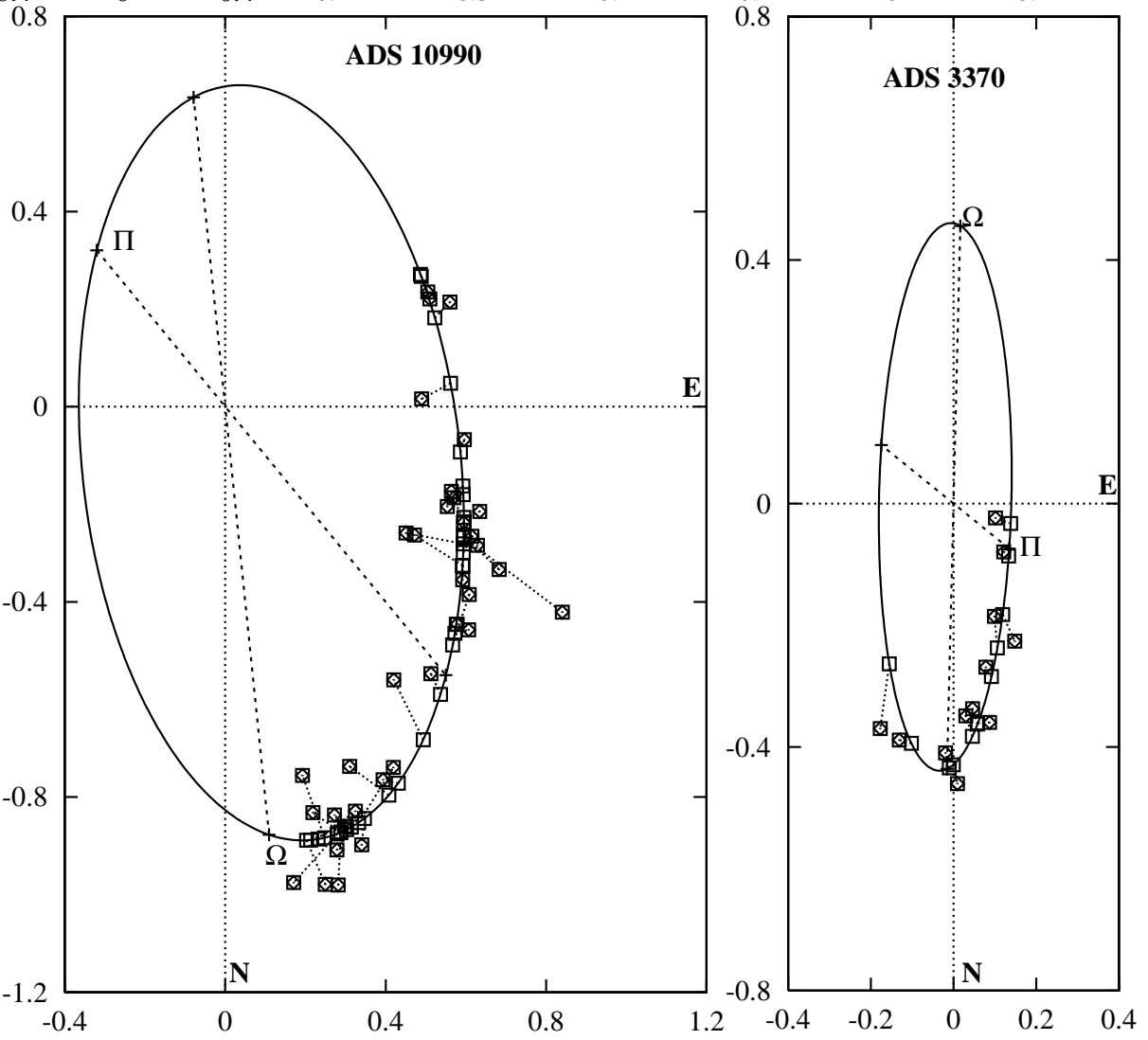

Fig. 1. Observed positions $(\otimes)$ and the ones emanating from the orbit calculation $(\square)$. The periastron position ( $\Pi)$ and the line of nodes $(\Omega)$ are given as well. The unit on both axes is second of arc 


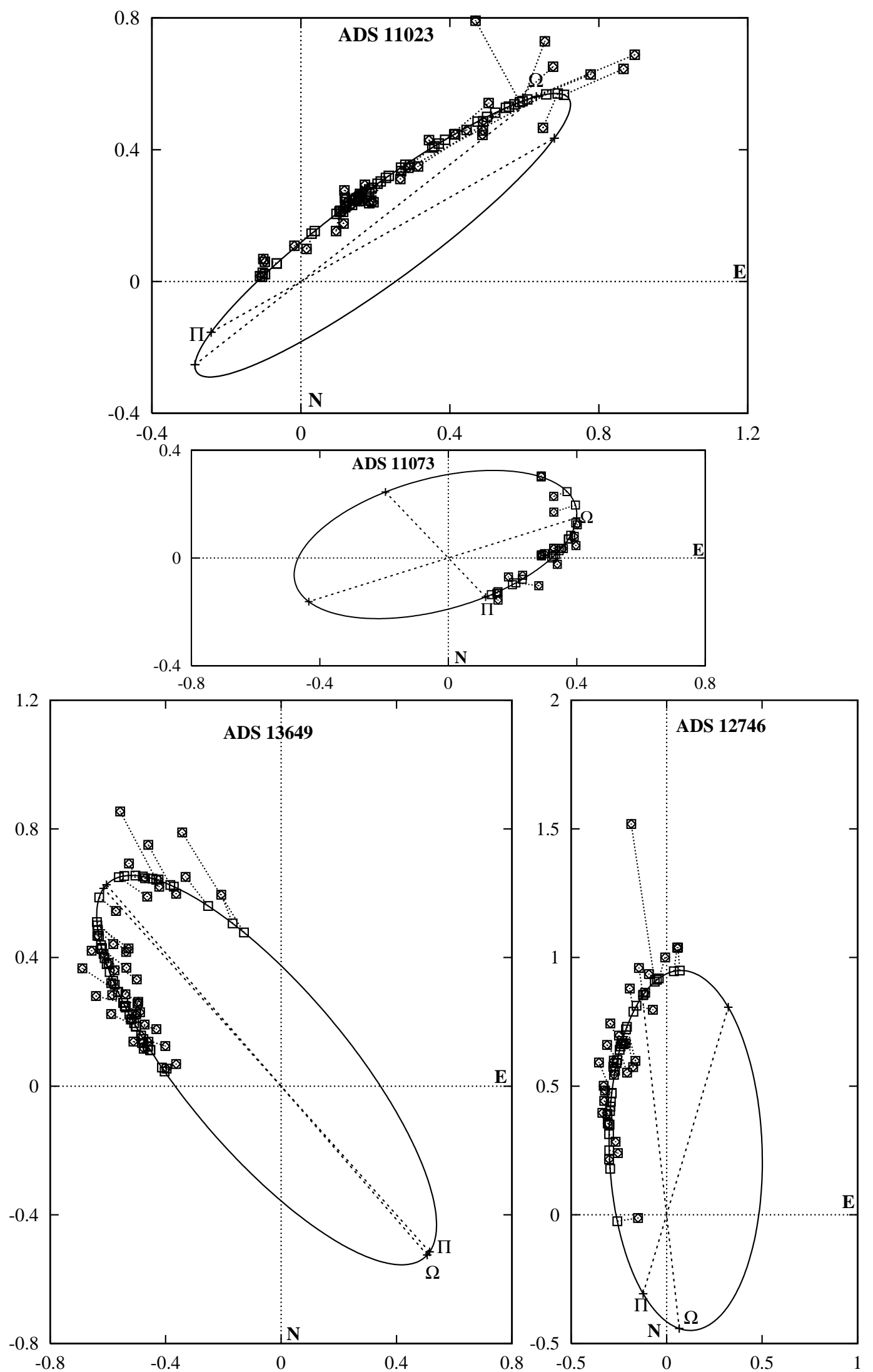

Fig. 1. continued 
Table 2. Measurements and $(\mathrm{O}-\mathrm{C})$

\begin{tabular}{lrcccrr}
\hline WDS 04089+4614 $=$ A & $998=$ ADS $3007=$ HD 25987 \\
\hline \multicolumn{1}{c}{$t$} & $\theta_{\mathrm{t}} \rho$ & $n$ & Obs. & $\Delta \theta$ & $\Delta \rho$ \\
\hline 1905.81 & 332.9 & $0^{\prime \prime} 33$ & 3 & $\mathrm{~A}$ & 1.75 & $-0^{\prime \prime} 07$ \\
1916.65 & 325.1 & 0.36 & 2 & $\mathrm{~A}$ & -1.20 & -0.04 \\
1932.84 & 318.9 & 0.38 & 2 & $\mathrm{~A}$ & 0.25 & 0.01 \\
1944.72 & 310.9 & 0.32 & 4 & Voûte & -1.17 & -0.02 \\
1946.35 & 311.4 & 0.42 & 6 & VBs & 0.34 & 0.08 \\
1947.93 & 309.2 & 0.36 & 4 & Bz & -0.84 & 0.03 \\
1955.68 & 300.5 & 0.31 & 4 & Bz & -4.01 & 0.01 \\
1961.85 & 295.3 & 0.29 & 1 & Cou & -3.90 & 0.01 \\
1962.816 & 299.3 & 0.24 & 4 & Bos & 1.03 & -0.03 \\
1962.945 & 298.2 & 0.26 & 4 & Wor & 0.05 & -0.01 \\
1964.10 & 300.0 & 0.26 & 1 & Cou & 3.00 & -0.01 \\
1965.06 & 110.3 & 0.37 & 4 & hz & -5.71 & 0.11 \\
1973.95 & 100.5 & 0.24 & 4 & hz & -4.40 & 0.02 \\
1976.837 & 280.8 & 0.23 & 4 & Wor & 0.49 & 0.02 \\
1978.88 & 273.7 & 0.18 & 3 & hz & -2.98 & -0.02 \\
1981.01 & 99.4 & 0.18 & 2 & Morel & 6.91 & -0.01 \\
1981.90 & 268.7 & 0.17 & 3 & hz & -1.91 & -0.01 \\
1982.06 & 97.9 & 0.17 & 3 & Cou & 7.64 & -0.01 \\
1983.0472 & 266.2 & 0.177 & 1 & McA87b & -1.86 & -0.00 \\
1983.0637 & 269.3 & 0.170 & 1 & McA87b & 1.28 & -0.01 \\
1983.84 & 85.7 & 0.18 & 2 & Cou & -0.51 & 0.01 \\
1984.0521 & 265.0 & 0.171 & 1 & McA87b & -0.70 & -0.00 \\
1985.00 & 83.7 & 0.17 & 2 & Cou & 0.34 & 0.00 \\
1985.8405 & 260.7 & 0.165 & 1 & McA87b & -0.50 & -0.00 \\
1986.8862 & 258.1 & 0.162 & 1 & McA89 & -0.28 & 0.00 \\
1987.7599 & 255.2 & 0.159 & 1 & McA89 & -0.70 & 0.00 \\
1989.7068 & 250.2 & 0.153 & 1 & Har92 & 0.19 & 0.00 \\
1989.813 & 249.8 & 0.14 & 1 & Cou, Ling & 0.13 & -0.01 \\
\hline & & & & & & \\
\hline & & & & & &
\end{tabular}

WDS $04275+1113=$ BU $1186=$ ADS $3228=$ HD 28217

\begin{tabular}{cccccrr}
\hline \multicolumn{1}{c}{$t$} & $\theta_{\mathrm{t}}$ & $\rho$ & $n$ & Obs. & $\Delta \theta$ & $\Delta \rho$ \\
\hline 1890.92 & 182.1 & $0^{\prime \prime} 59$ & 3 & $\beta$ & 0.71 & $0.0^{\prime \prime} 05$ \\
1893.61 & 185.3 & $0.55 \pm$ & 2 & Sp & 4.96 & 0.00 \\
1896.09 & 180.5 & 0.64 & 5 & Sp & 1.07 & 0.07 \\
1898.03 & 174.4 & 0.75 & 3 & A & -4.34 & 0.17 \\
1900.06 & 176.9 & 0.57 & 1 & See & -1.15 & -0.02 \\
1908.70 & 174.7 & 0.66 & 6 & Doo3, GrO3 & -0.65 & 0.04 \\
1922.60 & 167.4 & 0.58 & 2 & GrO & -4.03 & -0.07 \\
1936.65 & 166.2 & 0.62 & 4 & Bz & -1.27 & 0.00 \\
1943.74 & 168.5 & 0.61 & 3 & Voûte & 3.24 & 0.02 \\
1948.731 & 165.6 & 0.52 & 1 & VBs & 2.08 & -0.04 \\
1951.052 & 168.7 & 0.45 & 1 & Markowitz & 6.06 & -0.09 \\
1952.870 & 166.9 & 0.51 & 1 & Markowitz & 4.99 & -0.02 \\
1962.13 & 152.6 & 0.49 & 2 & Bz & -4.86 & 0.04 \\
1963.110 & 149.8 & 0.30 & 3 & Wor & -7.09 & -0.14 \\
1978.118 & 129.8 & 0.22 & 4 & Wor & -13.40 & -0.07 \\
1981.91 & 134.7 & 0.33 & 3 & hz & -2.24 & 0.08 \\
1983.0500 & 132.7 & 0.224 & 1 & McA et al. & -1.95 & -0.01 \\
1983.7162 & 132.3 & 0.213 & 1 & McA et al. & -0.90 & -0.02 \\
1984.0522 & 131.4 & 0.211 & 1 & McA et al. & -1.04 & -0.01 \\
1984.7072 & 130.4 & 0.206 & 1 & McA et al. & -0.49 & -0.01 \\
1984.9968 & 129.8 & 0.204 & 1 & McA et al. & -0.38 & -0.01 \\
1985.8488 & 127.6 & 0.196 & 1 & McA et al. & -0.37 & -0.01 \\
1986.8890 & 124.3 & 0.188 & 1 & McA et al. & -0.73 & -0.01 \\
1987.7600 & 122.5 & 0.181 & 1 & McA et al. & 0.14 & -0.01 \\
\hline & & & & & & \\
\hline
\end{tabular}

Table 2. continued

\begin{tabular}{|c|c|c|c|c|c|c|}
\hline \multicolumn{7}{|c|}{ WDS $04400+2301=$ HU $442=\operatorname{ADS} 3370=$ HD 29538} \\
\hline$t$ & $\theta_{\mathrm{t}}$ & $\rho$ & $n$ & Obs. & $\Delta \theta$ & $\Delta \rho$ \\
\hline 1901.86 & 333.8 & 0. & 3 & $\mathrm{Hu}$ & 4.90 & 0. \\
\hline 1921.33 & 340.9 & 0.41 & 3 & VBs & -4.13 & 0.00 \\
\hline 1944.76 & 357.0 & 0.41 & 4 & Voûte & -1.32 & -0.02 \\
\hline 1947.07 & 0.9 & 0.46 & 4 & BVs & 1.27 & 0.03 \\
\hline 1958.08 & 4.6 & 0.35 & 3 & Cou & -1.96 & -0.04 \\
\hline 1961.19 & 7.6 & 0.34 & 3 & $\mathrm{Bz}$ & -1.27 & -0.03 \\
\hline 1961.37 & 13.4 & 0.37 & 5 & $\mathrm{BVs}$ & 4.39 & 0.00 \\
\hline 1970.507 & 16.1 & 0.28 & 3 & Wor & -1.63 & -0.02 \\
\hline 1975.10 & 28.0 & 0.21 & 2 & Mul & 4.07 & -0.05 \\
\hline 1979.97 & 33.1 & 0.27 & 3 & $\mathrm{hz}$ & 0.13 & 0.05 \\
\hline 1987.7574 & 56.8 & 0.145 & 1 & $\mathrm{McA}$ et al & -0.25 & -0.01 \\
\hline 1991.9022 & 76.8 & 0.104 & 1 & McA et al & 0.18 & -0.04 \\
\hline
\end{tabular}

\begin{tabular}{|c|c|c|c|c|c|c|}
\hline$t$ & $\theta_{\mathrm{t}}$ & $\rho$ & $n$ & Obs. & $\Delta \theta$ & $\Delta \rho$ \\
\hline 1889.39 & 14.9 & $1^{\prime \prime} 01$ & 5 & $\beta$ & 1.43 & $0^{\prime \prime} 10$ \\
\hline 1890.47 & 19.70 & 0.91 & 3 & $\beta$ & 5.59 & 0.00 \\
\hline 1892.37 & 22.0 & 0.89 & 4 & $\beta$ & 6.77 & -0.03 \\
\hline 1894.13 & 14.9 & 0.78 & 4 & $\mathrm{Sp}$ & -1.37 & -0.14 \\
\hline 1897.51 & 10.5 & 0.99 & 3 & $\mathrm{~A}$ & -7.75 & 0.07 \\
\hline 1898.52 & 15.30 & 0.86 & 3 & $\beta$ & -3.54 & -0.06 \\
\hline 1898.53 & 16.6 & 1.02 & 3 & $\mathrm{~A}$ & -2.25 & 0.10 \\
\hline 1900.14 & 18.6 & 0.88 & 2 & Lewis & -1.19 & -0.04 \\
\hline 1901.49 & 17.6 & 0.95 & 3 & Doo & -2.99 & 0.03 \\
\hline 1903.67 & 30.1 & 0.85 & 1 & VBs & 8.23 & -0.06 \\
\hline 1905.39 & 21.3 & 0.96 & 4 & Doo & -1.59 & 0.05 \\
\hline 1913.21 & 23.3 & 0.80 & 3 & $\mathrm{~A} 2, \mathrm{GrO} 1$ & -4.32 & -0.09 \\
\hline 1916.50 & 27.7 & 0.86 & 2 & Fox & -1.98 & -0.02 \\
\hline 1926.48 & 37.3 & 0.70 & 1 & Fur & 1.01 & -0.14 \\
\hline 1935.14 & 43.5 & 0.75 & 4 & $\mathrm{Bz}$ & 0.83 & -0.05 \\
\hline 1943.46 & 58.0 & 0.72 & 6 & VBs & 8.42 & -0.03 \\
\hline 1945.32 & 53.3 & 0.76 & 3 & Voûte & 2.05 & 0.02 \\
\hline 1946.72 & 52.7 & 0.73 & 3 & $\mathrm{Bz}$ & 0.16 & 0.00 \\
\hline 1957.405 & 66.9 & 0.67 & 4 & Bos & 3.40 & 0.01 \\
\hline 1958.633 & 60.4 & 0.52 & 4 & Bos & -4.50 & -0.14 \\
\hline 1959.53 & 63.6 & 0.94 & 3 & Wor & -2.34 & 0.29 \\
\hline 1959.69 & 64.2 & 0.76 & 2 & Wor & -1.93 & 0.11 \\
\hline 1959.91 & 68.5 & 0.64 & 3 & VBs & 2.12 & -0.01 \\
\hline 1961.521 & 72.1 & 0.60 & 5 & Bos & 3.80 & -0.04 \\
\hline 1961.69 & 65.9 & 0.69 & 4 & $\mathrm{Bz}$ & -2.61 & 0.05 \\
\hline 1962.462 & $\operatorname{sim}]$ & iple & 1 & $\mathrm{Cou}$ & - & - \\
\hline 1965.51 & 73.1 & 0.59 & 3 & Cou & -0.19 & -0.03 \\
\hline 1966.69 & 69.9 & 0.59 & 4 & $\mathrm{Bz}$ & -4.93 & -0.02 \\
\hline 1971.342 & 83.7 & 0.60 & 4 & Wor & 2.53 & 0.01 \\
\hline 1980.603 & 92.0 & 0.49 & 4 & Wor & -3.01 & -0.07 \\
\hline
\end{tabular}


Table 2. continued

WDS $18033+3921=$ STF $2275=$ ADS $11023=$ HD 88432

\begin{tabular}{lrrlcrrr}
\hline \multicolumn{1}{c}{$t$} & $\theta_{\mathrm{t}}$ & $\rho$ & $n$ & Obs. & $\Delta \theta$ & $\Delta \rho$ \\
\hline 1832.20 & 127.9 & $1^{\prime \prime} 08$ & 3 & $\Sigma$ & -1.95 & 0.9 .17 \\
1844.35 & 126.8 & $0.8 \pm$ & 1 & Ma & -3.83 & -0.10 \\
1859.89 & 141.1 & 0.45 & 1 & Se & 9.43 & -0.42 \\
1878.59 & 128.4 & 1.13 & 1 & O $\Sigma$ & -4.64 & 0.31 \\
1882.54 & 138.9 & 0.98 & 1 & O $\Sigma$ & 5.54 & 0.17 \\
1884.50 & 150.2 & 0.92 & 5 & En & 16.68 & 0.12 \\
1888.64 & 138.8 & 0.47 & 2 & Sp & 4.93 & -0.32 \\
1892.60 & 134.7 & 0.94 & 2 & Big & 0.48 & 0.17 \\
1894.57 & 135.6 & 0.69 & 6 & Com & 1.20 & -0.07 \\
1901.46 & 133.1 & 0.66 & 3 & Lewis & -1.96 & -0.07 \\
1906.42 & 129.6 & 1.00 & 1 & Frm & -5.98 & 0.29 \\
1911.74 & 133.8 & 0.67 & 9 & GrO6, Neuj3 & -2.39 & -0.01 \\
1923.54 & 136.5 & 0.64 & 3 & Fur & -1.25 & 0.03 \\
1927.816 & 137.6 & 0.74 & 2 & G $\Sigma$ & -0.83 & 0.16 \\
1930.651 & 137.7 & 0.61 & 3 & G $\Sigma$ & -1.21 & 0.05 \\
1932.559 & 141.8 & 0.55 & 5 & G $\Sigma$ & 2.54 & 0.00 \\
1933.45 & 140.9 & 0.46 & 2 & VBs & 1.47 & -0.08 \\
1944.39 & 139.8 & 0.41 & 4 & Voute & -2.14 & -0.04 \\
1946.08 & 141.6 & 0.43 & 5 & VBs & -0.82 & -0.01 \\
1950.652 & 148.7 & 0.31 & 1 & Markowitz & 4.79 & -0.09 \\
1951.660 & 142.7 & 0.30 & 1 & Markowitz & -1.58 & -0.09 \\
1953.54 & 147.6 & 0.29 & 2 & VBs & 2.58 & -0.08 \\
\hline
\end{tabular}

WDS $18033+3921=$ STF $2275=$ ADS $11023=$ HD 88432

\begin{tabular}{|c|c|c|c|c|c|c|}
\hline$t$ & $\theta_{\mathrm{t}}$ & $\rho$ & $n$ & Obs. & $\Delta \theta$ & $\Delta \rho$ \\
\hline 1954.70 & 322.7 & 0.31 & 4 & $\mathrm{Bz}$ & -2.81 & -0.05 \\
\hline 1956.70 & 321.3 & 0.31 & 3 & $\mathrm{Bz}$ & -5.13 & -0.03 \\
\hline 1957.60 & 150.0 & 0.34 & 2 & VBs & 3.12 & 0.01 \\
\hline 1958.618 & 149.5 & 0.31 & 3 & Bos & 2.09 & -0.01 \\
\hline 1958.62 & 155.4 & 0.28 & 2 & VBs & 7.98 & -0.04 \\
\hline 1960.72 & 145.3 & 0.30 & 2 & $\mathrm{Bz}$ & -3.33 & -0.00 \\
\hline 1960.99 & 157.4 & 0.30 & 5 & VBs & 8.60 & -0.00 \\
\hline 1961.43 & 329.7 & 0.27 & 3 & Wor & 0.62 & -0.03 \\
\hline 1961.502 & $\sin \xi$ & gle & 2 & Dju & - & - \\
\hline 1962.102 & 151.5 & 0.27 & 4 & Bos & 1.97 & -0.02 \\
\hline 1942.49 & 152.1 & 0.24 & 3 & $\mathrm{Cou}$ & 2.30 & -0.05 \\
\hline 1962.71 & 153.5 & 0.27 & 3 & $\mathrm{Bz}$ & 3.55 & -0.01 \\
\hline 1965.72 & 152.3 & $0.26 e$ & 2 & $\mathrm{Bz}$ & -0.03 & 0.01 \\
\hline 1967.478 & 148.6 & 0.18 & 2 & Walker Jr. & -5.41 & -0.06 \\
\hline 1967.55 & 153.9 & 0.24 & 3 & Morel & -0.18 & 0.00 \\
\hline 1968.54 & 147.2 & 0.21 & 2 & $\mathrm{Cou}$ & -7.94 & -0.02 \\
\hline 1975.493 & 171.5 & 0.10 & 1 & Walker Jr. & 5.10 & -0.06 \\
\hline 1976.50 & 189.2 & 0.11 & 3 & $\mathrm{hz}$ & 20.31 & -0.04 \\
\hline 1987.529 & 238.3 & 0.114 & 1 & $\mathrm{C}$ & 8.52 & 0.03 \\
\hline 1987.529 & 236.0 & 0.122 & 1 & $\mathrm{~L}$ & 6.22 & 0.04 \\
\hline 1991.3192 & 255.4 & 0.106 & 1 & Har94 & -1.39 & 0.01 \\
\hline 1992.3132 & 266.6 & 0.111 & 1 & Har94 & -0.72 & 0.01 \\
\hline 1905.44 & $137: 6$ & $0 . \prime 42$ & 2 & Hu1, A1 & 0.31 & $0 .{ }^{\prime \prime} 00$ \\
\hline 1922.77 & 125.8 & 0.40 & 2 & VBs & 1.17 & -0.04 \\
\hline 1933.49 & 118.2 & 0.37 & 4 & VBs & 1.02 & -0.07 \\
\hline 1944.86 & 107.7 & 0.42 & 3 & VBs & -1.11 & 0.00 \\
\hline 1953.69 & 102.1 & 0.40 & 2 & Mul & 0.84 & 0.02 \\
\hline 1953.71 & 97.2 & 0.40 & 4 & $\mathrm{Bz}$ & -4.04 & 0.02 \\
\hline
\end{tabular}

Table 2. continued

\begin{tabular}{|c|c|c|c|c|c|c|c|}
\hline \multicolumn{8}{|c|}{ WDS $19389+3514=$ HU $953=\operatorname{ADS} 12746=$ HD 185696} \\
\hline$t$ & $\theta_{t}$ & $\rho$ & $n$ & Obs. & $\Delta$ & & $\Delta \rho$ \\
\hline 1958.552 & 96.7 & 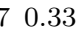 & 33 & Bos & 0.3 & & -0.02 \\
\hline 1958.63 & 92.0 & $\begin{array}{ll}0 & 0.29\end{array}$ & 9) 2 & VBs & -4.2 & & -0.06 \\
\hline 1959.78 & 96.8 & $\begin{array}{ll}8 & 0.33\end{array}$ & 32 & Cou & 1.8 & & -0.02 \\
\hline 1961.71 & 86.5 & 50.34 & $4 \quad 4$ & $\mathrm{Bz}$ & -6.2 & & 0.01 \\
\hline 1963.526 & 93.5 & 50.30 & 4 & Wor & 3.0 & & -0.02 \\
\hline 1974.524 & 74.8 & 80.24 & $4 \quad 4$ & Wor & 3.4 & & 0.00 \\
\hline 1976.49 & 70.2 & 20.30 & 4 & Mul & 3.5 & & 0.07 \\
\hline 1977.54 & 69.7 & $\begin{array}{ll}7 & 0.20\end{array}$ & 3 & $\mathrm{hz}$ & 5.8 & & -0.02 \\
\hline 1981.653 & 49.4 & $\begin{array}{ll}40.20 \\
-15\end{array}$ & 3 & Wor & -1.8 & & 0.00 \\
\hline 1983.50 & 44.9 & 90.22 & 23 & Mul & 0.2 & & 0.03 \\
\hline 1904.47 & 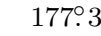 & $\begin{array}{lll}3 & 1 . \prime\end{array}$ & 43 & $\mathrm{Hu}$ & 0.8 & & $0 ! \prime 09$ \\
\hline 1909.46 & 177.5 & 51.04 & 45 & Bow2, Dob3 & -0.7 & & 0.09 \\
\hline 1922.09 & 180.9 & 91.00 & 2 & VBs & -2.1 & & 0.08 \\
\hline 1923.69 & 186.1 & 10.94 & 43 & Chan1, Plq2 & 2.4 & & 0.03 \\
\hline 1925.55 & 187.4 & 41.53 & 31 & Schem & 3.0 & & 0.62 \\
\hline 1935.36 & 187.8 & $\begin{array}{ll}8 & 0.87\end{array}$ & 73 & $\mathrm{Bz}$ & -0.7 & & 0.01 \\
\hline 1935.68 & 189.0 & $\begin{array}{ll}0 & 0.97\end{array}$ & 72 & VBs & 0.3 & & 0.11 \\
\hline 1941.555 & $5 \quad 185.5$ & 50.80 & 1 & Arend & -5.8 & & -0.03 \\
\hline 1944.42 & 192.7 & 70.90 & 3 & Voute & -0.0 & & 0.09 \\
\hline 1950.751 & 195.6 & $\begin{array}{ll}6 & 0.62\end{array}$ & 21 & Markowitz & -0.5 & & -0.14 \\
\hline 1951.706 & 197.3 & 30.60 & 1 & Markowitz & 0.5 & & -0.15 \\
\hline 1955.796 & 198.09 & 90.70 & 6 & Rabe & -1.1 & & -0.01 \\
\hline 1956.54 & 200.0 & $\begin{array}{ll}0 & 0.74\end{array}$ & 43 & Bos & 0.2 & & 0.03 \\
\hline 1956.78 & 199.0 & $\begin{array}{ll}0 & 0.70\end{array}$ & 3 & $\mathrm{Bz}$ & -0.9 & & -0.01 \\
\hline 1957.60 & 201.9 & $\begin{array}{ll}9 & 0.80\end{array}$ & 3 & VBs & 1.4 & & 0.10 \\
\hline 1958.684 & $\begin{array}{ll}4 & 200.8\end{array}$ & $\begin{array}{ll}8 & 0.59\end{array}$ & 92 & Dju, Dac & -0.4 & & -0.10 \\
\hline 1961.727 & $7 \quad 205.5$ & $\begin{array}{ll}5 & 0.73\end{array}$ & $\begin{array}{ll}3 & 3\end{array}$ & Wor & 2.0 & & 0.07 \\
\hline 1962.60 & 207.1 & 10.61 & 13 & Cou & 2.9 & & -0.04 \\
\hline 1962.66 & 205.0 & $\begin{array}{lll}0 & 0.66\end{array}$ & $6 \quad 5$ & VBs & 0.8 & & 0.01 \\
\hline 1964.78 & 206.1 & 10.64 & $4 \quad 4$ & $\mathrm{hz}$ & 0.1 & & 0.02 \\
\hline 1970.472 & 211.1 & $\begin{array}{lll}1 & 0.69\end{array}$ & $\begin{array}{ll}9 & 1\end{array}$ & Ole & -0.3 & & 0.14 \\
\hline 1970.552 & 213.5 & $\begin{array}{ll}50.60 \\
\end{array}$ & b 2 & Pop & 1.9 & & 0.05 \\
\hline 1972.473 & $\begin{array}{l}3 \\
3\end{array}$ & $\begin{array}{ll}9 & 0.58\end{array}$ & $\begin{array}{ll}8 & 1\end{array}$ & Ole & 0.1 & & 0.05 \\
\hline 1973.628 & 216.6 & $\begin{array}{ll}6 & 0.55\end{array}$ & $5 \quad 1$ & Pop & 1.4 & & 0.04 \\
\hline 1974.636 & $5 \quad 218.4$ & $\begin{array}{ll}40.50 \\
\end{array}$ & D 4 & Wor & 1.9 & & -0.00 \\
\hline 1977.55 & 221.1 & $\begin{array}{lll}1 & 0.47\end{array}$ & 73 & $\mathrm{hz}$ & 0.4 & & 0.01 \\
\hline 1977.556 & 220.5 & $\begin{array}{ll}5 & 0.52\end{array}$ & 22 & Holden & -0.1 & & 0.06 \\
\hline 1979.52 & 223.4 & $\begin{array}{ll}4 & 0.39\end{array}$ & $\begin{array}{ll}9 & 3 \\
\end{array}$ & $\mathrm{hz}$ & -0.5 & & -0.04 \\
\hline 1982.721 & L 226.9 & $\begin{array}{ll}9 & 0.35\end{array}$ & 53 & Massone & -3.3 & & -0.04 \\
\hline 1986.216 & 234.5 & $\begin{array}{ll}5 & 0.37\end{array}$ & 72 & $\mathrm{Zul}$ & -4.4 & & 0.02 \\
\hline 1995.62 & 274.7 & 70.15 & 53 & $\mathrm{hz}$ & -0.8 & & -0.11 \\
\hline WDS 201 & $176+262$ & - & & $4=\mathrm{AI}$ & 12640 & NOW & 1 \\
\hline$t$ & $\theta_{\mathrm{t}}$ & $\rho$ & $n$ & Obs. & & $\Delta \theta$ & $\Delta \rho$ \\
\hline 1880.47 & $204.1 \mathrm{c}$ & $0 ! \prime 86$ & 2 & $\beta$ & & $8: 43$ & $0 !^{\prime \prime} 37$ \\
\hline 1883.70 & $199.8 \mathrm{C}$ & 0.63 & 3 & Ho & & 0.83 & 0.10 \\
\hline 1888.49 & 203.1 & & 1 & $\mathrm{Lv}$ & & 0.01 & - \\
\hline 1890.85 & 207.5 & 0.73 & 7 & Sp & & 2.66 & 0.12 \\
\hline 1901.78 & 211.8 & 0.70 & 2 & A & & 0.38 & -0.02 \\
\hline 1903.02 & 212.0 & 0.88 & 4 & Doo & & -0.05 & 0.15 \\
\hline 1908.10 & 213.61 & 1.02 & 2 & Moth & & -0.85 & 0.25 \\
\hline 1909.55 & 213.90 & 0.77 & 15 & Dob3, Lv2, Gr & $\mathrm{rO} 10$ & -1.19 & -0.01 \\
\hline 1913.43 & $214.7 \mathrm{c}$ & 0.75 & 14 & Fox $3, \mathrm{GrO} 1$ & 11 & -2.03 & -0.06 \\
\hline 1916.68 & $217.7 \mathrm{c}$ & 0.87 & 3 & Ptt & & -0.33 & 0.04 \\
\hline
\end{tabular}


Table 2. continued

\begin{tabular}{|c|c|c|c|c|c|c|}
\hline \multicolumn{7}{|c|}{ WDS $20176+2622=$ BU $984=$ ADS $13649=$ HD 193095} \\
\hline$t$ & $\theta_{\mathrm{t}}$ & $\rho$ & $n$ & Obs. & $\Delta \theta$ & $\Delta \rho$ \\
\hline 1922.24 & 216.5 & 0.80 & 8 & Lv5, GrO3 & -3.65 & -0.05 \\
\hline 1925.28 & 218.6 & 0.75 & 17 & Lv3, Bail2, Plq2, Berm10 & -2.66 & -0.11 \\
\hline 1942.37 & 226.7 & 0.79 & 3 & Voûte & -0.64 & -0.07 \\
\hline 1953.56 & 232.4 & 0.68 & 2 & VBs & 0.83 & -0.14 \\
\hline 1954.87 & 231.2 & 0.68 & 2 & Mul & -0.90 & -0.13 \\
\hline 1958.73 & 233.9 & 0.79 & 4 & $\mathrm{hz}$ & 0.17 & 0.00 \\
\hline 1961.82 & 236.4 & 0.74 & 4 & $\mathrm{hz}$ & 1.29 & -0.02 \\
\hline 1962.74 & 237.5 & 0.78 & 4 & $\mathrm{Bz}$ & 1.96 & 0.02 \\
\hline 1965.955 & 236.6 & 0.60 & 4 & Walker Jr. & -0.50 & -0.13 \\
\hline 1970.116 & 241.6 & 0.67 & 3 & Wor & 2.28 & -0.02 \\
\hline 1972.63 & 242.8 & 0.56 & 4 & $\mathrm{hz}$ & 2.02 & -0.11 \\
\hline 1973.677 & 244.3 & 0.65 & 1 & Ole & 2.88 & -0.01 \\
\hline 1975.648 & 242.2 & 0.61 & 3 & Holden & -0.49 & -0.03 \\
\hline 1975.72 & 242.1 & 0.78 & 1 & Zul & -0.64 & 0.14 \\
\hline 1978.57 & 246.5 & 0.70 & 1 & Zul & 1.77 & 0.09 \\
\hline 1978.570 & 242.2 & 0.56 & 1 & Pop & -2.53 & -0.05 \\
\hline 1979.355 & 244.9 & 0.54 & 4 & Walker Jr. & -0.42 & -0.06 \\
\hline 1979.54 & 246.0 & 0.55 & 3 & $\mathrm{hz}$ & 0.54 & -0.04 \\
\hline 1979.817 & 248.4 & 0.56 & 4 & Wor & 2.73 & -0.03 \\
\hline 1981.64 & 246.1 & 0.55 & 2 & $\mathrm{hz}$ & -1.03 & -0.02 \\
\hline 1982.804 & 248.0 & 0.51 & 1 & Massone & -0.12 & -0.05 \\
\hline 1983.860 & 247.75 & 0.467 & 3 & Scardia & -1.30 & -0.08 \\
\hline 1984.631 & 249.2 & 0.63 & 4 & $\mathrm{Zul}$ & -0.56 & 0.09 \\
\hline 1984.719 & 252.1 & 0.51 & 3 & Wor & 2.25 & -0.03 \\
\hline 1987.81 & 252.8 & 0.42 & 3 & Beau & -0.17 & -0.08 \\
\hline 1989.636 & 254.5 & 0.50 & 1 & Doc & -0.54 & 0.02 \\
\hline 1989.636 & 254.9 & 0.53 & 1 & Lin & -0.14 & 0.05 \\
\hline 1990.553 & 253.3 & 0.48 & 1 & Pri & -2.85 & 0.01 \\
\hline 1990.553 & 256.3 & 0.49 & 1 & Lap & 0.15 & 0.02 \\
\hline 1994.80 & 262.2 & 0.40 & 3 & hz & 0.15 & -0.02 \\
\hline
\end{tabular}

At the time of periastron passage in 2012 the component separation will be $\sim 0$ ". 3 .

WDS 18054+6216 = ADS $11073=$ HU $1290=$ HD 166206 The elements are obtained from a positionangle change exceeding $90^{\circ}$.

The value obtained for the absolute magnitude and the F8 spectrum indicates that the system belongs to subgiants (LC IV). The orbital parallax obtained here is 0 .'008. The agreement with the trigonometric one following from the Hipparcos Catalogue $\left(0{ }^{\prime \prime} 00779 \pm 0 . \prime 00075\right)$ is complete.

WDS 19389+3514 = ADS $12746=$ HU $953=$ HD 185696 This pair grows in its measuring suitability and the component separation at the time of intersecting the node line will be $\sim 0^{\prime \prime} .3$. The obtained orbital parallax is $0 . \prime 013$ whereas in the Tycho Catalogue one finds $0.029 \pm 0$. 006 .

WDS 20176+2622 $=$ ADS $13649=$ BU $984=$ HD 193095 The significant number of measurements for this pair, in spite of a modest change of its position angle $-60^{\circ}$, permits a good orbit determination.
Table 3. Ephemerides

\begin{tabular}{|c|c|c|c|c|c|c|c|c|}
\hline \multirow{2}{*}{$\begin{array}{c}\text { WDS } \\
\text { ADS } \\
t\end{array}$} & \multicolumn{2}{|c|}{$\begin{array}{c}04089+4614 \\
3007\end{array}$} & \multicolumn{2}{|c|}{$\begin{array}{c}04275+1113 \\
3228\end{array}$} & \multicolumn{2}{|c|}{$\begin{array}{c}04400+2301 \\
3370\end{array}$} & \multicolumn{2}{|c|}{$\begin{array}{c}18018+0118 \\
10990\end{array}$} \\
\hline & $\theta_{\mathrm{t}}$ & $\rho$ & $\theta_{\mathrm{t}}$ & $\rho$ & $\theta_{\mathrm{t}}$ & $\rho$ & $\theta_{\mathrm{t}}$ & $\rho$ \\
\hline 1999.0 & $214^{\circ} 37$ & 0. & $70 \div 49$ & $0^{\prime \prime} 14$ & $113^{\circ} 00$ & $0 .{ }^{\prime \prime} 15$ & $123^{\circ} 46$ & $0^{\prime \prime} 56$ \\
\hline 2000.0 & 209.84 & 0.13 & 65.45 & 0.15 & 117.33 & 0.16 & 125.03 & 0.56 \\
\hline 2001.0 & 205.19 & 0.12 & 60.66 & 0.15 & 121.35 & 0.16 & 126.58 & 0.57 \\
\hline 2002.0 & 200.38 & 0.12 & 56.15 & 0.16 & 125.08 & 0.17 & 128.12 & 0.57 \\
\hline 2003.0 & 195.40 & 0.12 & 51.95 & 0.16 & 128.53 & 0.18 & 129.65 & 0.57 \\
\hline 2004.0 & 190.19 & 0.12 & 48.07 & 0.17 & 131.69 & 0.19 & 131.17 & 0.57 \\
\hline 2005.0 & 184.68 & 0.11 & 44.50 & 0.18 & 134.61 & 0.19 & 132.67 & 0.58 \\
\hline 2006.0 & 178.72 & 0.11 & 41.23 & 0.19 & 137.29 & 0.20 & 134.15 & 0.58 \\
\hline 2007.0 & 172.07 & 0.10 & 38.23 & 0.19 & 139.76 & 0.21 & 135.62 & 0.58 \\
\hline 2008.0 & 164.36 & 0.09 & 35.49 & 0.20 & 142.04 & 0.22 & 137.08 & 0.58 \\
\hline
\end{tabular}

\begin{tabular}{ccccccccc}
\hline WDS & $18033+3921$ & $18054+6216$ & $19389+3514$ & $20176+2622$ \\
ADS & 11023 & 11073 & 12746 & 13649 \\
\hline & & & & & & & & \\
\hline$t$ & $\theta_{\mathrm{t}}$ & $\rho$ & $\theta_{\mathrm{t}}$ & $\rho$ & $\theta_{\mathrm{t}}$ & $\rho$ & $\theta_{\mathrm{t}}$ & $\rho$ \\
\hline
\end{tabular}

$1999.0 \quad 285.44 \quad 0^{\prime \prime} .16 \quad 344.71 \quad 0^{\prime \prime} 21 \quad 292.89 \quad 0^{\prime \prime} 25 \quad 2699^{\circ} 30 \quad 0^{\prime \prime} 37$ $\begin{array}{llllllllll}2000.0 & 287.52 & 0.17 & 341.82 & 0.22 & 298.02 & 0.26 & 271.33 & 0.36\end{array}$ $\begin{array}{lllllllll}2001.0 & 289.39 & 0.18 & 339.10 & 0.22 & 303.05 & 0.26 & 273.48 & 0.35\end{array}$ $\begin{array}{llllllllll}2002.0 & 291.07 & 0.19 & 336.53 & 0.23 & 307.93 & 0.26 & 275.75 & 0.34\end{array}$ $\begin{array}{llllllllll}2003.0 & 292.59 & 0.20 & 334.11 & 0.24 & 312.61 & 0.27 & 278.16 & 0.33\end{array}$ $\begin{array}{llllllllll}2004.0 & 293.98 & 0.21 & 331.83 & 0.25 & 317.08 & 0.28 & 280.71 & 0.32\end{array}$ $\begin{array}{llllllllll}2005.0 & 295.24 & 0.22 & 329.67 & 0.25 & 321.31 & 0.29 & 283.39 & 0.31\end{array}$ $\begin{array}{lllllllll}2006.0 & 296.41 & 0.23 & 327.64 & 0.26 & 325.31 & 0.29 & 286.23 & 0.31\end{array}$ $\begin{array}{llllllllll}2007.0 & 297.48 & 0.24 & 325.72 & 0.27 & 329.07 & 0.30 & 289.20 & 0.30\end{array}$ $\begin{array}{llllllllll}2008.0 & 298.47 & 0.25 & 323.90 & 0.28 & 332.60 & 0.31 & 292.32 & 0.29\end{array}$

The parallax and the absolute magnitude are calculated following the empirical mass-luminosity relation concerning the HR main sequence. According to the absolute magnitude from the Tycho Catalogue this pair would be a subgiant (LC IV). Our parallax $\left(00^{\prime \prime} 015\right)$ is within the parallax-error limits indicated by the Tycho Catalogue $(0.011 \pm 0 . \prime 005)$.

\section{References}

Angelov T., 1993, Bull. Obs. Astron. Belgrade 148, 1

Eichhorn H.K., Xu Yu-lin, 1990, ApJ 358, 575

ESA, 1997, The Hipparcos and Tycho Catalogue

McAlister H.A., Hartkopf W.I., 1988, CHARA Contribution 2,1

Popović G.M., Pavlović R., 1995, Bull. Obs. Astron. Belgrade 152,55

Worley C.E., Douglass G.G., 1996, The Washington Double Star Catalog, 1996.0, available on cdsarc.u-strasbg.fr 